Dear Editor,

\title{
A note on D-policy bulk queueing systems
}

We offer new studies of the queueing process of $D$-policy models and correct results of [2].

\section{Preliminaries}

In [2], the $D$-policy did not apply to the queueing process in the general case. The results of [2] are corrected by applying [1] and [3]. Throughout, we use the notation of [2].

Customers enter the system in accordance with a bulk Poisson input of intensity $\lambda$ and with $a(z)$ as the probability generating function of arriving batches. They are serviced singly in accordance with the i.i.d. random variables $\Sigma_{1}, \Sigma_{2}, \ldots$ with the joint probability density function $B(x)$ and Laplace-Stieltjes transform $\sigma(\theta)$. When the system is exhausted, service is suspended with the server staying idle in the system, leaving the system for multiple vacation trips or a single vacation trip. Each suspension mode lasts until the system's workload becomes $D$ or greater at one of the 'observation epochs'. Let $\tau=\left(\tau_{0}, \tau_{1}, \ldots\right)$ be the sequence of such observation epochs, $X_{0}, X_{1}, \ldots$ be the increments of units' replenishment over $\tau$, and $Y_{0}, Y_{1}, \ldots$ be the respective increments of the workload. With $v=\inf \left\{k: B_{k}=Y_{0}+\cdots+Y_{k}>D\right\}$, $\tau_{v}$ is the first passage time. The observed value $B_{v}$ of $\left\{B_{k}\right\}$ at $\tau_{v}$ is the workload, and $A_{v}$ is the queue length (where $A_{k}=X_{0}+\cdots+X_{k}$ ) at $\tau_{v}$ (the first excess level projection of $B_{v}$ onto $\left.\left\{A_{k}\right\}[1],[2]\right)$.

Now, at the beginning of the first busy period after suspension, the server starts servicing one virtual customer whose service time is $B_{v}$, so that $Q_{n}=Q\left(T_{n}\right), n=0,1, \ldots$, where $T_{1}=\Sigma_{1}$, if $Q_{0}>0$, and $T_{1}=\tau_{v}+B_{\nu}$, if $Q_{0}=0$. The embedded chain is that of an $M / G / 1$ queue with bulk input and start-up time. We need [2]:

$$
\begin{aligned}
L(z, \vartheta, \theta) & :=\mathbb{E}\left[z^{A_{v}} \mathrm{e}^{-\vartheta B_{v}} \mathrm{e}^{-\theta \tau_{v}}\right] \\
& =\gamma_{0}(z, \vartheta, \theta)-[1-\gamma(z, \vartheta, \theta)] \mathcal{L}_{s}\left\{\frac{\gamma_{0}(z, \vartheta+s, \theta)}{1-\gamma(z, \vartheta+s, \theta)}\right\}(D),
\end{aligned}
$$

where

$$
\begin{gathered}
\gamma_{0}(z, \vartheta, \theta)=\mathbb{E}\left[z^{X_{0}} \mathrm{e}^{-\vartheta Y_{0}} \mathrm{e}^{-\theta \tau_{0}}\right], \quad \gamma(z, \vartheta, \theta)=\mathbb{E}\left[z^{X_{1}} \mathrm{e}^{-\vartheta Y_{1}} \mathrm{e}^{-\theta \chi_{1}}\right], \\
\chi_{n}=\tau_{n}-\tau_{n-1}, \quad n=1,2, \ldots, \quad \text { and } \quad \mathcal{L}_{s} F(x)=\operatorname{Lapl}^{-1}\left(\frac{1}{s} F(s)\right)(x), \quad x \geq 0 .
\end{gathered}
$$

Multiple vacations.

$$
\tau_{0}=X_{0}=Y_{0}=0, \quad \gamma_{0}=1
$$

and

$$
\begin{array}{rlrl}
\gamma(z, \vartheta, \theta)=\mathbb{E}\left[z^{X_{1}} \mathrm{e}^{-\vartheta Y_{1}} \mathrm{e}^{-\theta \chi_{1}}\right] & =\gamma(\lambda(z \sigma(\vartheta), \theta)), \\
\sigma(\vartheta)=\mathbb{E}\left[\mathrm{e}^{-\vartheta \Sigma_{1}}\right], & \lambda(z, \theta) & =\lambda-\lambda a(z)+\theta, \\
a(z)=\mathbb{E}\left[z^{U_{1}}\right], & \gamma(\theta) & =\mathbb{E}\left[\mathrm{e}^{-\theta \chi_{1}}\right] .
\end{array}
$$

Received 23 November 1999; revision received 27 November 2000. 
(The latter is the Laplace-Stieltjes transform of a one vacation trip.) Consequently,

$$
L(z, \vartheta, \theta)=1-[1-\gamma(\lambda(z \sigma(\vartheta), \theta))] \mathscr{L}_{s}\left\{\frac{1}{1-\gamma(\lambda(z \sigma(\vartheta+s), \theta))}\right\}(D) .
$$

Dormant server.

$$
\gamma(z, \vartheta, \theta)=\frac{\lambda}{\lambda+\theta} a(z \sigma(\vartheta))
$$

yielding

$$
L(z, \vartheta, \theta)=1-\left[1-\frac{\lambda}{\lambda+\theta} a(z \sigma(\vartheta))\right] \mathscr{L}_{s}\left\{\frac{1}{1-(\lambda /(\lambda+\theta)) a(z \sigma(\vartheta+s))}\right\}(D) .
$$

Single vacation.

$$
\begin{aligned}
& \gamma_{0}(z, \vartheta, \theta)=\gamma(\lambda(z \sigma(\vartheta), \theta)), \\
& \gamma(z, \vartheta, \theta)=\frac{\lambda}{\lambda+\theta} a(z \sigma(\vartheta)),
\end{aligned}
$$

$$
\begin{aligned}
L(z, \vartheta, \theta)= & \gamma(\lambda(z \sigma(\vartheta), \theta)) \\
& -\left[1-\frac{\lambda}{\lambda+\theta} a(z \sigma(\vartheta))\right] \mathscr{L}_{s}\left\{\frac{\gamma(\lambda(z \sigma(\vartheta+s), \theta))}{1-(\lambda /(\lambda+\theta)) a(z \sigma(\vartheta+s))}\right\}(D) .
\end{aligned}
$$

The corresponding formulas for the marginal functional $\mathscr{B}(\vartheta)=\mathbb{E}\left[\mathrm{e}^{-\vartheta B_{v}}\right]$ will read as follows.

Multiple vacations.

$$
\mathscr{B}(\vartheta)=1-[1-\gamma(\lambda(\sigma(\vartheta)))] \mathscr{L}_{s}\left\{\frac{1}{1-\gamma(\lambda(\sigma(\vartheta+s)))}\right\}(D),
$$

where $\lambda(z)=\lambda(z, 0)$.

\section{Dormant server.}

$$
\mathcal{B}(\vartheta)=1-[1-a(\sigma(\vartheta))] \mathcal{L}_{s}\left\{\frac{1}{1-a(\sigma(\vartheta+s))}\right\}(D) .
$$

\section{Single vacation.}

$$
\mathscr{B}(\vartheta)=\gamma(\lambda(\sigma(\vartheta)))-[1-a(\sigma(\vartheta))] \mathcal{L}_{s}\left\{\frac{\gamma(\lambda(\sigma(\vartheta+s)))}{1-a(\sigma(\vartheta+s))}\right\}(D) .
$$

Now, the probability generating function $p(z)$ of the invariant probability measure $p$ of the embedded queueing process is:

$$
p(z)=p_{0} \frac{z \mathscr{B}(\lambda(z))-\sigma(\lambda(z))}{z-\sigma(\lambda(z))}, \quad p_{0}=\frac{1+\overline{\mathcal{B}} \lambda a-\rho}{1-\rho},
$$

where $\rho=\lambda a S, a=\mathbb{E}\left[U_{1}\right], S=\mathbb{E}\left[\Sigma_{1}\right]$, and $\overline{\mathcal{B}}=\rho \bar{\tau}$, with $\bar{\tau}=\mathbb{E}\left[\tau_{v}\right]$. 


\section{Continuous time parameter process}

The limiting distribution $\pi=\left(\pi_{0}, \pi_{1}, \ldots\right)$ of $Q(t)$ exists given $\rho<1$ and is sought in the form of its probability generating function $\pi(z)=(1 / C) p h(z)$, where $C$ is given below, and $h(z)=\left(h_{i}(z) ; i=0,1, \ldots\right)^{\top}$ with the probability generating functions of the respective rows of the integrated (over $\mathbb{R}_{+}$) semi-regenerative kernel

$$
K(t)=\left\{K_{i k}(t)=\mathbb{P}^{i}\left\{Q(t)=k, T_{1}>t\right\} ; i, k=0,1, \ldots\right\}
$$

being given by

$$
h_{i}(z)=\sum_{k=0}^{\infty} z^{k} \int_{0}^{\infty} K_{i k}(t) \mathrm{d} t=z^{i} \Delta(z), \quad i>0,
$$

where

$$
\Delta(z)=\frac{1-\sigma(\lambda(z))}{\lambda(z)} .
$$

Theorem. ([3].)

$$
T(z, \theta)=\int_{0}^{\infty} \mathrm{e}^{-\theta t} \mathbb{E}\left[z^{N_{t}} \mathbf{1}_{\left\{\tau_{v}+B_{v}>t\right\}}\right] \mathrm{d} t=\frac{1-L(z, \lambda(z, \theta), \theta)}{\lambda(z, \theta)},
$$

where L satisfies (1.1), (1.3), (1.4) or (1.5) and $\lambda(z, \theta)$ is defined in (1.2).

In our case,

$$
h_{0}(z)=T(z, \theta)=\int_{0}^{\infty} \mathbb{E}\left[z^{N_{t}} \mathbf{1}_{\left\{\tau_{v}+\Sigma>t\right\}}\right] \mathrm{d} t=\frac{1-L(z, \lambda(z), 0)}{\lambda(z)},
$$

with the following variants of $L$.

\section{Multiple vacations.}

$$
L(z, \lambda(z), 0)=1-[1-\gamma\{\lambda[z \sigma(\lambda(z))]\}] \mathcal{L}_{s}\left\{\frac{1}{1-\gamma\{\lambda(z \sigma(\lambda(z)+s))\}}\right\}(D) .
$$

\section{Dormant server.}

$$
L(z, \vartheta, 0)=1-[1-a\{z \sigma(\lambda(z))\}] \mathscr{L}_{s}\left\{\frac{1}{1-a\{z \sigma(\lambda(z)+s)\}}\right\}(D) .
$$

\section{Single vacation.}

$$
L(z, \vartheta, 0)=\gamma\{\lambda[z \sigma(\lambda(z))]\}-[1-a\{z \sigma(\lambda(z))\}] \mathcal{L}_{s}\left\{\frac{\gamma\{\lambda[z \sigma(\lambda(z)+s)]\}}{1-a\{z \sigma(\lambda(z)+s)\}}\right\}(D) .
$$

From (2.1)-(2.3) we get

$$
p h(z)=\Delta(z) p(z)+p_{0} \frac{\sigma(\lambda(z))-L(z, \lambda(z), 0)}{\lambda(z)} .
$$

The mean stationary value of the service cycle is equal to $C=p c$, where

$$
c=\left(c_{i}=\mathbb{E}^{i}\left[T_{1}\right] ; i=0,1, \ldots\right)^{\top}, \quad c_{0}=\bar{\tau}(1+\rho), \quad c_{i}=S, \quad i>0,
$$

and $\bar{\tau}=\mathbb{E}\left[\tau_{\nu}\right]$. This yields

$$
C=p_{0}(\bar{\tau}(1+\rho)-S)+S .
$$

Finally,

$$
\pi(z)=\frac{1}{C} p h(z)
$$




\section{References}

[1] Dshalalow, J. H. (1997). On the level crossing of multi-dimensional delayed renewal processes. J. Appl. Math. Stoch. Anal. 10, 355-361.

[2] Dshalalow, J. H. (1998). Queueing processes in bulk systems under the D-policy. J. Appl. Prob. 35, 976-989.

[3] Dshalalow, J. H. (2001). Time dependent analysis of multivariate marked renewal processes. Submitted.

Yours sincerely,

Jewgeni H. Dshalalow

Department of Applied Mathematics,

Florida Institute of Technology,

Melbourne, FL 32901,

USA.

Email address: eugene_d@bellsouth.net 